\title{
Testicular metastasis from adenocarcinoma of the prostate
}

\author{
HAL J. BASHEIN, DO \\ PHILLIP GINSBERG, DO \\ JOHN R. ZOND, DO \\ LEONARD H. FINKELSTEIN, DO
}

Reported are two cases of prostate cancer metastatic to the testicle. Both patients underwent bilateral orchiectomies for treatment of symptomatic metastatic prostate cancer. Testicular metastasis was found incidentally in one patient and was suspected in the other. This site of spread is rare. The clinical significance and prognosis are discussed and the pertinent literature reviewed.

(Key words: Prostate cancer, metastasis, testicular cancer)

Nonlymphomatous secondary tumors of the testicle are rare. A total of 209 cases of secondary testicular tumors have been reported in the literature since the first case of renal cell carcinoma metastatic to the testis was reported by Creevy in $1935 .{ }^{1}$ Seamans ${ }^{2}$ reported the first case of carcinoma that metastasized from the prostate to the testis in 1938.

Only 72 cases of prostatic cancer with metastasis to the testicle have been reported in the English medical literature. Most of these cases are found at autopsy or as incidental findings after orchiectomy for hormonal management of metastatic prostate cancer. In a re-

From the Division of Urology, Department of Surgery, Osteopathic Medical Center of Philadelphia (Drs Bashein, Ginsberg, and Finkelstein) and the Department of Pathology and Laboratory Medicine, Philadelphia College of Osteopathic Medicine (Dr Zond), Philadelphia, $\mathrm{Pa}$.

Reprint requests to Hal J. Bashein, DO, 9207-B Jamison Ave, Philadelphia, PA 19115. view of approximately 24,000 autopsies, there were 15 cases of metastatic involvement of the testis, an incidence of $0.06 \% .^{3}$ A testicular mass is the initial symptom in only a small group of patients, and patients are usually older than 60 years. We report two cases of secondary testicular tumors from prostate carcinoma (one of which was diagnosed preoperatively) in which special histologic staining for prostate-specific antigen (PSA) or prostatic acid phosphatase (PAP) was diagnostic.

\section{Case 1}

In 1979, a 70-year-old man had adenocarcinoma of the prostate staged as B1 after pelvic lymphadenectomy. Transurethral prostatectomy was performed, and external beam radiation therapy (EBRT) was administered. Recurrent tumor at the vesical neck was resected 8 years later. Prostatespecific antigen levels increased steadily to $34 \mathrm{ng} /$ mL. However, repeated bone scans consistently showed no abnormality. Examination of the external genitalia in 1989 revealed a $1.5 \times 1 \times 1.5-\mathrm{cm}$ mass on the superior pole of the left testis (Fig 1). Bilateral orchiectomy was performed, and results of immunoperoxidase staining for PSA and PAP were strongly positive (Fig 2). The final diagnosis was metastatic prostate adenocarcinoma of the left testicle. The disease has not yet progressed.

\section{Case 2}

A 68-year-old man had obstructing prostatism. Subsequent transurethral prostatectomy revealed poorly differentiated adenocarcinoma of the prostate with invasion into the urinary bladder. A computed tomography scan showed widespread pelvic 


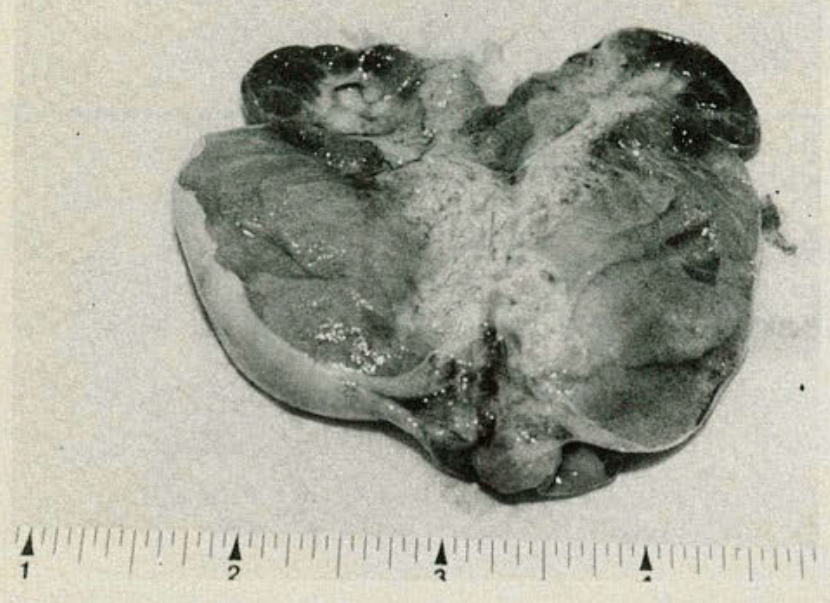

Figure 1. Gross specimen shows metastatic tumor.

and para-aortic adenopathy. Back pain rapidly ensued, and a bone scan showed abnormalities at several sites. Bilateral orchiectomy for androgen deprivation was performed on palpably normal testicles. Histologic examination revealed a focus of poorly differentiated, metastatic prostatic adenocarcinoma in the left testicle. Further attempts at palliation by administration of pelvic EBRT therapy and flutamide proved futile. The patient died 3 months after initial diagnosis.

\section{Discussion}

Carcinoma of the prostate metastatic to the testicle is more common in men 60 years of age and older. However, $20 \%$ to $40 \%$ of the cases occur in patients younger than 40 years. ${ }^{4,5}$ Only $6 \%$ of all patients initially have a testicular mass leading to an occult primary tumor. Bilateral testicular metastases from all histologic types is $15 \%$. However, the prostatic primary tumor accounts for $75 \%$ of bilateral metastases. Serum $\alpha$-fetoprotein (AFP) and human chorionic gonadotropin (HCG) are occasionally elevated in patients with nontesticular primary tumors, but markedly elevated levels in young patients suggest a nonseminomatous germ-cell tumor.

Many tumors have been reported to involve the testes by metastasis, the most common being prostate tumor (35\%). Other common tumors include pulmonary $(17 \%)$, colon $(8 \%)$, melanoma $(8 \%)$, and kidney $(6 \%)$. Other malignancies reported in the literature to have metastasized to the testis are those of the stomach ( 5 cases), pancreas (4 cases), nervous system (neuroblastoma, 4 cases), bladder ( 3 cases), rectum ( 2 cases), penis ( 2 cases), and 1 case each of carcinoid, retinoblastoma, cylindroma, and mesothelioma as well as cancers of the thyroid, ureter, bile duct, liver, salivary gland, and appendix. ${ }^{6-8}$

The gross and microscopic pathologic features of metastatic disease in the testis are highly variable. Many cases, especially those diagnosed at autopsy, have no gross lesion and are detected only at microscopic review. Clinically diagnosed testicular metastases are generally associated with testicular enlargement and with intratesticular nodules or masses replacing the testicular parenchyma. The microscopic features vary and reflect the site of origin of the metastasis.

Microscopic foci of poorly differentiated, metastatic prostate carcinoma detected in orchiectomy specimens can be diagnostically challenging. Immunoperoxidase staining for PSA and PAP can be of value. In addition, noting the lipofuscin pigment found in interstitial cells will assist in the differential diagnosis. Features commonly associated with metastatic lesions of the testis are predominant proliferation in the interstitium and vascular invasion. Appropriate application of stain for epithelial mucin and immunoperoxidase stains for AFP and HCG may be helpful.

The route of dissemination of metastatic tumors in the testis has been the focus of much discussion. There are four possible routes: arterial embolism, retrograde venous dissemination, retrograde lymphatic spread, and intraductal spread by means of the vas deferens. The demonstration of communicating lymphatics draining the prostate, testis, and epididymis provides the anatomic basis for metastatic prostatic carcinoma in the testis. Luminal involvement of the vas deferens suggests that retrograde spread by this route underlies testicular metastases. ${ }^{9}$

Prostate cancer is the most common cancer affecting men in the United States and the third leading cause of cancer deaths in men. The American Cancer Society estimated 122,000 new cases of prostate cancer and 


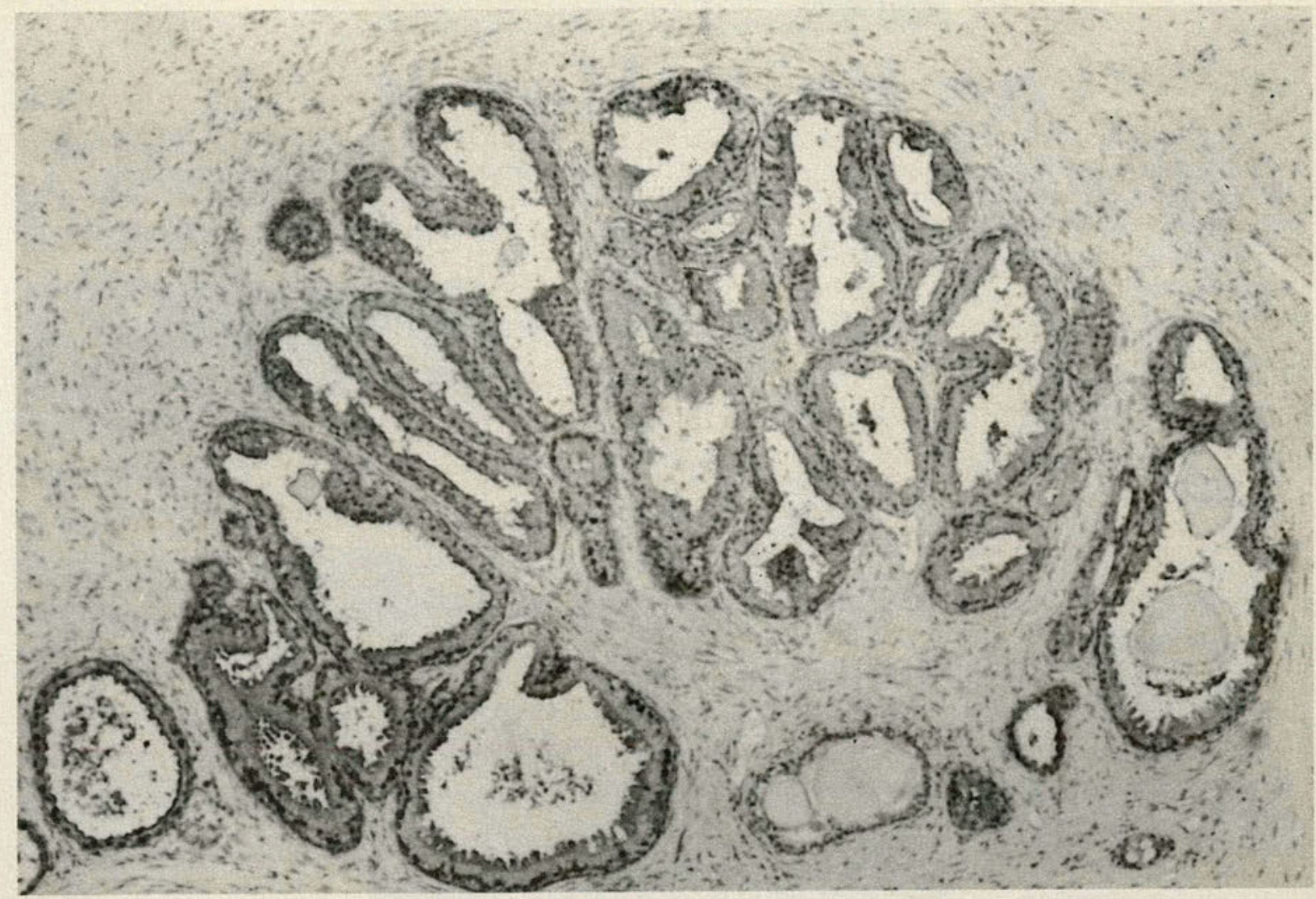

Figure 2. Metastatic tumor from prostate. The tumor cells stain positively for prostatic acid phosphatase (PAP) when the immunoperoxidase method is used (original magnification $\times 100$ ).

32,200 deaths as a result of the disease in $1991 .^{10}$ Men younger than 50 years have a higher incidence of metastatic disease at the time of clinical diagnosis. ${ }^{9}$ Although less than $1 \%$ of patients with clinically detectable prostate cancer are younger than 50 years, it is imperative to rule out a prostate malignancy when a patient has a testicular mass.

Adenocarcinoma of the prostate can spread to nearly every tissue. Despite its frequency and capacity for wide dissemination, its appearance in the testis is rare. None of the many explanations postulated for this phenomenon has been proved. Inadequate examination of the testis during postmortem studies may also contribute to the paucity of cases. Perhaps more cases will be confirmed by using immunoperoxidase staining techniques for PSA.

The prognosis is not unfavorable; survival is apparently not influenced by the patient's age, duration of the prostate carcinoma, or whether the testicular metastasis was clini- cally manifest or detected on microscopic examination. ${ }^{11}$

1. Creevy CD: Confusing clinical manifestations of malignant renal neoplasms. Arch Intern Med 1935;55:895-898.

2. Seamans JH: Carcinoma of the prostate with metastasis to the testis. J Urol 1938;40:524-528.

3. Pienkos EJ, Jablokow UR: Secondary testicular tumors. Cancer 1972;30:481-486.

4. Price EB Jr, Mostofi FK: Secondary carcinoma of the testis Cancer 1957;10:592-600.

5. Hanash KE, Carney JA, Kelalis PP: Metastatic tumors to the testicles: Routes of metastasis. J Urol 1969;102:465-469.

6. Patel SR, Richardson RL, Krols L: Metastatic cancer to the testis: A report of 20 cases and review of the literature. $J$ Urol 1989;142:1003-1005.

7. Finkelstein LH, Manfrey SJ, Arsht DB, et al: Penile metastases from intraductal carcinoma of prostate diagnosed by corpora cavernosa aspiration biopsy. JAOA 1983;82:549-550.

8. Fiorelli RL, Finkelstein LH, Fernandes JJ: Metastasis of prostate gland adenocarcinoma to penile and scrotal cutaneous tissues. JAOA 1989;89:349-351.

9. Peterson O: Urologic Pathology. Philadelphia, JB Lippincott Co, 1986, pp 522-524, 625-635.

10. Silverberg E, Lubera JA: Cancer statistics, 1991. CA 1991;41:28-29.

11. Weitzner S: Survival of patients with secondary carcinoma of prostate in the testis. Cancer 1973;32:447-449. 


\section{The Falls Has It All}

Halfway between Chicago and New York is Cuyahoga Falls General Hospital in Cuyahoga Falls, Ohio. Put your medical skills to work at a modern facility without the high pressures of a metropolis. Enjoy the entertainment and recreational amenities of a big city, with rural and suburban settings only minutes from where you work. You'll find that our community hospital provides a desirable environment for your practice, while the surrounding community provides a desirable environment for you and your family, complete with hundreds of fine restaurants and several major shopping malls.

Major Universities

- The University of Akron

- Kent State University

Major Sports

- Cleveland Indians Baseball

- Cleveland Browns Football

- Cleveland Cavaliers Basketball

- Cleveland Crunch Soccer

Major Museums

- Akron Art Museum

- Cleveland Museum of Art

- Cleveland Museum of Natural History

- National Inventors' Hall of Fame

- Pro Football Hall of Fame
Major Events

- The Soap Box Derby

- The World Series of Golf

- PBA Tournament of Champions

- The Cleveland Budwieser 500

Major Entertainment

- The Ohio Ballet

-E. J. Thomas Performing Arts Hall

- Blossom Music Center

(Summer Home of the Cleveland Orchestra)

- Sea World of Ohio

- Geauga Lake Amusement Park

- Cedar Point Amusement Park

- The Cuyahoga Valley National Recreation Area

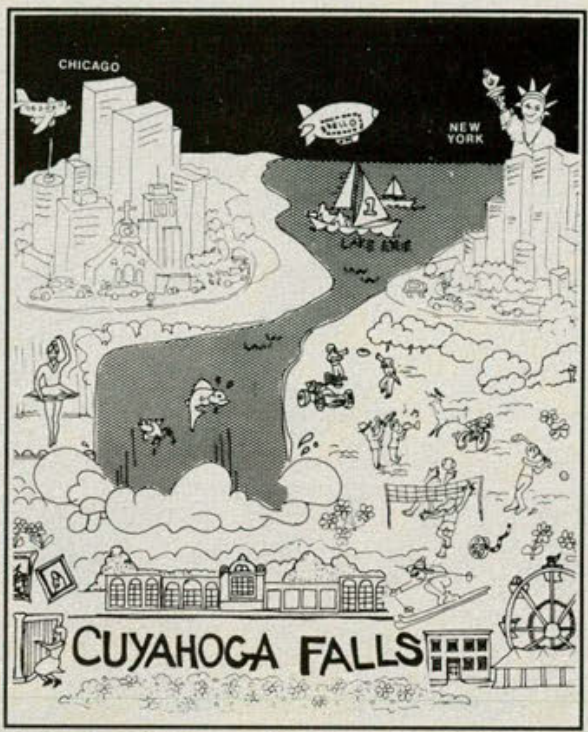

Approved for 17 Internships and 26 Residency Programs

- Obstetrics •Internal Medicine •Emergency •Dermatology

-Surgery •Anesthesiology

-Orthopedic •General Practice

\section{(216) 971-7224}
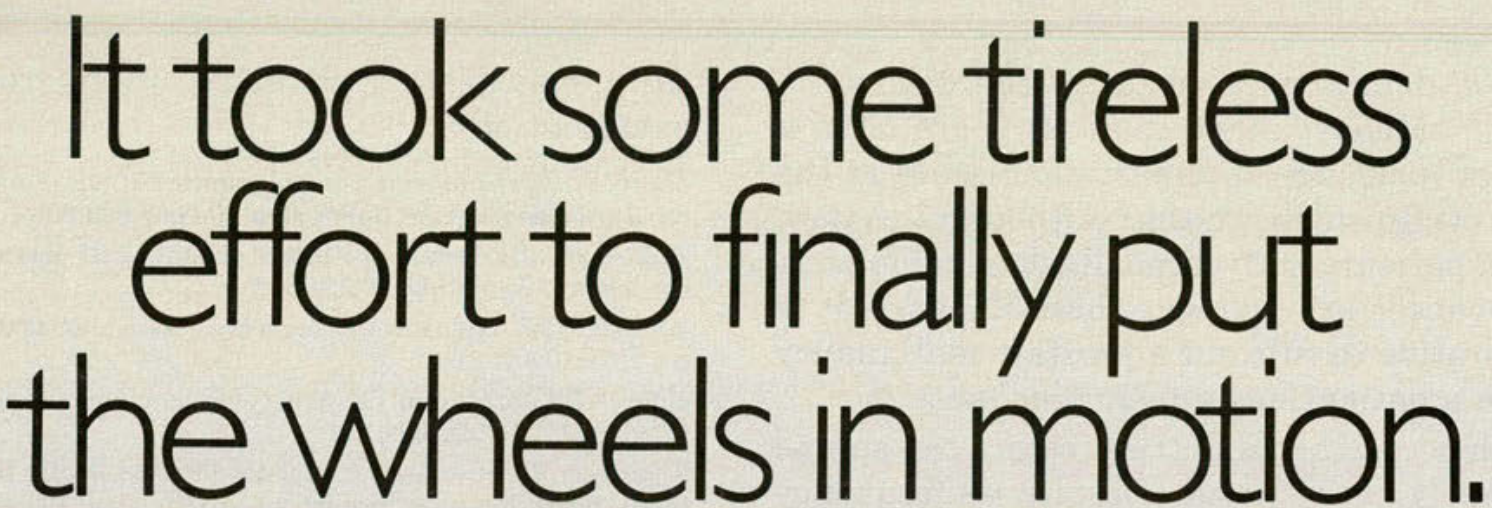
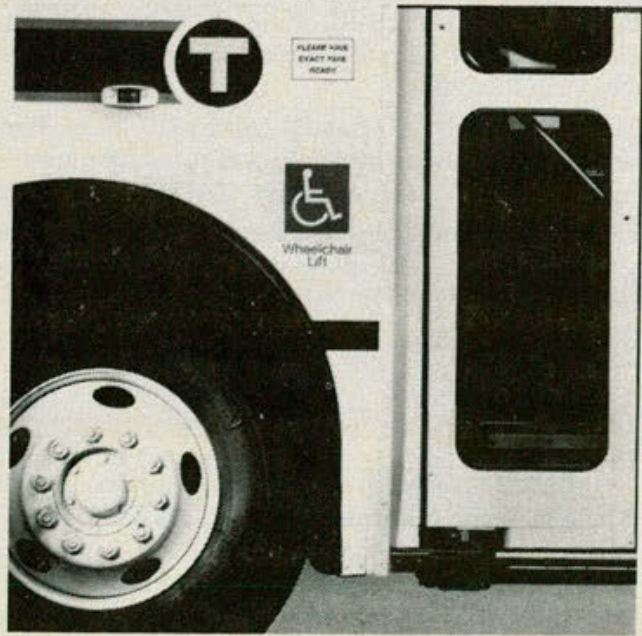

No one would argue that people with disabilities deserve equal access to public transportation. But it took your support to bring the issue to the fore. And the Americans with Disabilities Act to finally make it law. Thank you.

Because public transportation is for everyone.

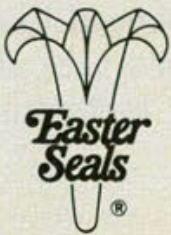

